\title{
The Development of Quality Assurance Mechanism and Accreditation of Open and Distance Education in Pakistan
}

\author{
Waqar Un Nisa Faizi', Anila Fatima Shakil², Rabia Abdul Karim³ \\ ${ }^{1}$ Assistant Professor, Shaheed Banazir Bhutto Women University, Peshawar \\ ${ }^{2}$ Associate Professor, Jinnah University for Women Karachi, Karachi \\ ${ }^{3}$ Associate Professor, Jinnah University for women Karachi, Karachi
}

\section{A B S T R A C T}

The research was conducted with the intent to analyze authorization and quality development procedure for the open Distant Education system that is adopted in Pakistan. The Higher Education Commission in Pakistan has implemented various strategies for assuring a high level of quality education is provided. One of the efforts includes reorganizing the infrastructure of the educational institutes for its proficient implementation. The research method adopted in it is of qualitative nature which constitutes of analyzing various literature and evaluating the responses of questions gathered from the 21 Heads of Department. The responses were also generated from the four public universities in which the randomly selected answers were of forty students. The responses were studied to evaluate their perception, prospection, and the difficulties they perceive are associated with the methods and services adopted for improving educational quality in Pakistan. The study suggests that the quality enhancement follows an outline which is based on the fundamentals of making students nurture on the international viewpoint, educational quality standards and ownership standards, self-determination and relations, improving the student's knowledge quality and integrating all the parties associated for the formation of an effective system.

KEYWORDS: Quality Assurance, Accreditation, Distance Education

\section{INTRODUCTION}

In the recent times, the educational sector is considered one of the most rapidly growing sectors across the globe. The growth is evident from the increasing student's statistics in institutes and across regions along the commuting of students for higher education purpose from one place to another. The increases in demands for education have created difficulties for the educational management in terms of transparency and 
accountability (Hallak, 2006). Various occupations have changed their hiring practices and now hire those individuals who possess a university degree. In order to gain power and position in a reputable or prestige organization, students are required to hold certification from the university. This increasing demand for education has become a challenge to manage among the all-time learners and adults (Daniel, 1996). The increasing demand serves both as an advantage and disadvantage as it increased student's nurturance opportunities while it also paves way for the corrupt practices in the institution of higher education.

The Open and distance learning (ODL) programs offered in Asian and Pacific regions comprise of distinctive attributes as the majority of the student population is involved in it. For instance, the numbers of institutes that provide open and distant learning in Asia and Pacific are seven which include STOU University, UT University, PNU University, IGNOU University, AIOU University, KNOU University, the Anadolu University, and the CCRTVU University in Thailand, Indonesia, Iran, India, Pakistan, Korea, Turkey, and China respectively. According to Jung (2005), the students who participated in the year 2004 in these programs were 5.6. The term Mega University is defined as the institute which offers distance education to more than one hundred thousand individuals (Daniel, 1996).

The quality enrichment tool is adopted for improving the standard of the education that is provided in the higher education institutes and universities along with their commitment. The improvement of quality in education constitutes developing, devising and applying a system that is autonomous and uses compelling strategies, which improves the quality of the education gradually (Leimaitre, 2008).

The nation-derived objective for the education involves devising a system that is based on quality, as reported by the US Department of Education in 2002. On a basis of this, the institute can guarantee that the services or goods provided meet the set criteria for education. This set criterion is met by the organization by explaining the context in which the term quality is used, by defining the process which will keep the quality characteristics intact, and by elucidating on the confined lines in which the quality will be tested for meeting the standardized goals. The quality assurance procedure is not only 
confined to the parameters of improving it but also assuring that the projected aims and goals are fulfilled through it (Glossary, 2010). This serves as the baseline for the analysis of the practices and the functions performed in the tertiary organization by an outside body and are regarded as Higher Education certification. The certification is only allotted when the standard outlined fulfilled by the agency (Lem, 1992).

The information by Nordic Recognition Information Centre (2006) provides that in 2002, the higher education commission (HEC) replaced the grant commission of university in Pakistan, which was responsible for sustaining a standardized level of quality education across the country. The mechanism adopted for the achievement of it's include outlining the objectives at each level of the post-secondary education. It also facilitated the institute by providing them grants and funds for marinating the set standard of education. The sustainment and enhancement of the established education standards in education is the obligation of the HEC. The vital element of involved in all includes maintenance of quality learning for students. Along with other various responsibilities of the HEC, the founding of the quality education and certification provision system falls in it too.

The HEC has not only confined its responsibility for the establishment of the quality assurance mechanism, but it also includes the improvement of the learning of students in the post-secondary level in the country. The obligations, that fall in this includes the establishment of the programs for a four-year timeframe to the bracket of undergraduate students along with the formulation of the developmental courses in other programs which are being provided. HEC is also responsible for monitoring and continual revision of the course at all stage of higher education. Moreover, it also offers the programs for improving the faculty competence, which is ensured by appointing individuals who possess a degree of Ph.D. HEC also develops various scholarship programs in the country and abroad as well for students to assist them in the attainment of the Ph.D. degree. For this, they have started offering various courses for the newly appointed teachers, which goes by the name of refresher courses. The timeframe set for developing the fundamental concepts of teaching in these courses in three months. The argument presented by the Nordic Recognition Information Centers (2006) states that 
these programs which promote international academicians for conducting research and teaching play an important part in the enhancement of the education that is provided in the institutes.

\section{Statement of the problem}

According to the argument presented by Stella and Gnanam (2004), the upsurge of the distance learning as a form of education as well as it changing context and meaning with time, along with its divergent nature in contrast to the traditional paradigm of learning, and its influence across the globe has resulted in increased difficulty in measuring the quality of the education provided. The responsibility for providing authorization to the educational institutes across the world is taken by the Distance Education and Training Council (DETC) that is situated in the US. According to the research of the DETC (1998), it was discovered that the countries who are associated with the DETC as members have strong conviction that the regulation benefits is enhanced when the new activities are introduced by the new providers of education along with their contribution in the increased difficulty for quality assessors (DETC, 1998). The issues concerning the quality of the education have increased because of educational enhancement of its promotional activities which are regarded as the trade. The World Health Organization (WHO) has also endorsed the commercialization of the education sector for the provision of higher education. This has led the World Trade Organization considerations concerning the General Agreement on Trade in Services (GATs).

By the year 1998, the nations which had been involved in the GATs in Services were 21. However, in the present time, the increasing number of countries of signing GAT in services is centered on eradicating the obstacles placed in the intercontinental exchange of education (Marginson, 2002). With these activities and practices at the rise, the tension concerning the commerce in the market environment tends to take higher education into its tentacles. This gathered up a perplexing compilation of various options provided in the market for learning, which leads the consumers to believe that quality asserting agents are set to provide more material concerning the quality education services which will assist them in making decisions. The Open and Distance education courses have been criticized for offering poor quality education. The criticism emerge 
because the divergence that prevails in the offered courses in the program with the normal programs which further mitigates the standards of the students who are enrolled in it. Another aspect of criticizing it is because it hinders the growth and jeopardized the mechanism of post-secondary education which is implemented in Pakistan. This makes it imperative for studying the quality assurance and certification standard of the higher educational institutions.

\section{Research Objectives}

The study is conducted with the following objectives

- To study the concerns associated with the performance of the quality assurance in Pakistan as well as the policy which are executed in higher education institution by it.

- To evaluate the viewpoint of the professors, educational receivers and the departmental heads concerning the quality enhancement and assurance in Pakistan.

- To find out the expectations which are associated with the higher education quality and assurance system implemented in Pakistan

\section{Research questions}

- To learn the practices adopted by the universities for reviewing, examining and enhancing the quality of education.

- To study students and faculty perception of the students concerning the quality and assurance in the education of the university they are part of

- To learn about the departmental heads and students perception about the education standards and its quality assurance in the federal universities of Pakistan.

- To evaluate the impact of the current practices, policies, infrastructure along with the policy formulators attitudes, their values and convictions have on the Pakistani universities educational policies concerning quality improvement and quality sustainment. 


\section{The significance of the Study}

The research conducted and the survey results obtained will be beneficial for the higher educational institutes in the country as well as the individuals who devise policies for the maintenance of educational quality and its improvement. The finding of the research will serve as the baseline for the system which is responsible for the quality maintenance in the system as well as assists in strategizing the procedure for the distance learning institutions which will ensure high-quality education provision. Moreover, the research can serve as the initial point where the higher education commission can devise program and procedures which can be implemented in the formal and distance education programs. Also considering the further studies concerning the aspects related to stakeholders and more, the findings of the study can supply in the valuable information for the makers of the policy and assist them in devising a policy which is effective and efficient in improving the educational quality in institutes of Pakistan while also assuring it.

\section{LITERATURE REVIEW}

\section{Quality Assurance}

The term quality assurance refers to a procedure which functions keeping in consideration the fundamentals of providing standardized services products to meet the quality criteria set. The governmental bodies formulate the standards along with the institutes concerning the particular industry values. The main motive of the quality assurance is to provide quality goods and services without having detrimental effects in the process on individual and environment alike (Inglis, 2005).

The fundamentals which are taken into consideration for ensuring the delivery of quality education in the higher learning institutions are as follows (Bureau, 2009):

1. First are the culture and the structure which the institute constitutes of. The institution has the obligation for the provision of quality education programs as well as learning. The institution is required to adopt a valuable culture as well as quality implementation system. This requires the top management high competency level in terms of delivering quality, which makes it imperative to set a quality framework which is smart in nature i.e. specific, measurable, attainable, 
realistic, and time-bound. Moreover, the participation of the stakeholders in the procedure is compulsory for forming a culture that assures quality. The structure should also supply assistance for improving the program effectiveness and efficacy pertaining to quality

2. To balance the self-governing and accountability aspect of the institution. This highlights the fact that the institution must be nurtured on the accountability factors which he holds towards the public although they are autonomous in operation. For this, the institutes should keep the societal values in high regard and allow the external bodies to review the quality improvement and assurance system adopted. In addition, the implemented quality standards by institute must have been developed by studying the standardized one and in correspondence to the discussion with the stakeholders

3. It also involved forming a cohesion of institutes mission with the quality assurance i.e. to set the standards for the quality which emphasize on the fulfilment institution general mission.

4. The quality enhancement aspect constitutes the institution to make continual efforts for the enhancement of the standards set which are in correspondence with the demands of the society through its programs. The program should be monitored from time to time for modifying it according to the developmental needs of the society.

5. The qualitative and quantitative data integrated into the system pertaining to quality involved reviewing the gathered data that points towards the institution status and serves as a framework for decision making. So the institutes are required to gather data and evaluate it for generating information like the student's graduation rate, the progression of staff pertaining to academics and how funds are managed

6. The fundamental of peer analysis and self-evaluation includes assessment to take place at every stage for deriving a conclusion which states whether the goals formulated have been achieved or not. This assessment is said to highlight the strong and weak points and assist in determining the corrective measures which 
are to be taken for enhancing the quality through practices. This assessment will assist in evaluating the quality level.

7. The involvement of the external bodies is basically done so that concerns on the institution quality are highlighted which provide the various suggestion and course of action for improving the program quality. The external bodies are involved in evaluating the student's performance level and what difference exists in their performance in contrast to the other institutes

8. The transparency in the policies pertaining to quality involves that the masses must be aware of the policy such as they must know the program details. It also involved attaining the stakeholder's feedback for the mechanism implemented for the maintenance and sustainment of quality.

\section{Quality Education and its Assurance:}

The main aim of the quality assurance in higher education is to supply in the support for the expansion, and development of the nation. The developing nations in the world have been adamant on reinforcing a system which provides high-quality education for its future generations. For this purpose, it has devised a quality assurance infrastructure in various institutes by creating quality assurance department. The senior officer in the quality assurance department is allocated with all the functionality powers. A structure is devised which is to be followed for formulating the programs, as well as getting approval. In many countries, various commissions have been set up to supervise the quality issue that occurs in the institutions (Brunetto \& Farr-Wharton, 2005; Mukhopadhyay, 2005).

Although, the formation of such a system is faced with many difficulties in which main problems include the quality, quantity, and equity. Even though these occur in nature, a proper structural set is required for subsiding it in the most beneficial way. This consideration led to the modifications in the higher education which include the advent of Higher Education Commission in Pakistan as an autonomous body under the presidential ordinance of 2002. This commission is responsible for devising and implementing plans which improve, analyze, and evaluate the education system as well as innovate its functioning (Khawaja, 1996). 
The Higher Education Commission recognized the most pertinent issues under the five-year plan also called Medium Term Development Framework, which pointed towards the enhancement of the quality in terms of the focus, and vital task in the Pakistans' post-secondary learning (Higher Education Commission, 2009).

The higher education system in Pakistan has undergone substantial modifications. These changes were made by forming a curriculum plan which elucidates on the standards of the program as well as duration such as the end of Bachelors programs of two years in 14 years of educations as well as the end Bachelor of Science degree of four years after the 16 years of education. Various financial and capital measures have been provided to the institutes for improving the standards for conducting research as well as the competency of the teaching staff. Since the establishment of the HEC, the number of educational institute's has increased. Now, 124 public universities are present comprising of two distance universities along with 57 private universities who deliver educational services to more than 170 million students. The institutes which are privately owned follow the outline standards set by HEC for ensuring the provision of quality education as it the sole body for recognizing the educational degree worth and the certifying the institution (Higher Education Commission, 2009).

\section{Standing of QEC and Present Concerns in Universities of Pakistan:}

The United States Department of Education (2002) monitors the goals and objectives set by the nations which are to be met by the deliverance of quality education. The goals and objectives are determined on the nation's capacity of excellence, along with social excellence level and the educational success. The educational quality improvement determines the commitment level of the higher educational institutes for the quality as reported in the study of Lemaitre (2008). It also involves its ability for the formulation of the strategies in the education systems which are automatically regulated for improving the educational quality. The concern regarding quality is not a sole aspect but it integrates the production and efforts for the achieving excellence in its system by working hard (Rauf, 2004).

The framework of achieving standardized education serves as the baseline for the civilized nation for improving the higher education, as it is the main source from which 
the nation's progress is determined. The quality in the education sector serves as the catalyst for the country progress; the countries that realize the significance of the quality in education system take all the necessary measures for its up gradation. This quality serves as the basis for the HEC ranking of the universities. Through this, the university applies the set standards of quality education to achieve a high level of quality in education, though the real struggle in its implementation is the lack of the conferred staff.

According to the study of Mohanthy (2000), the culture of the society serves as the basis on the for the higher education in the country which amplifies the staff commitment particularly of those employees which are determined on improving the skill set and learning by conducting research with this aim. Moreover, the study of Husain (2007), illustrates that the fathoming results are reliable, therefore, the instructor of the university is expected to undergo a quality research. The Higher Education Commission is making significant efforts for improving the educational institution infrastructure. The alteration in the infrastructure is improving the strategies which are implemented for the quality assurance in learning as well as in research and teaching activities

The HEC is making every single attempt at improving the country quality education standards in order to meet the international quality criteria of education. With this motive in mind, HEC has devised a well-defined strategy. The well-defined strategy is centered on assuring quality in education and improvement that follows a consistent pattern in post-secondary education across the country (Batool \& Qureshi, 2006).

Many initiatives both short and long-term are devised by HEC for the enhancing the standard of the learning that takes place at university as well as another educational level. It has been supplied in that the HEC has made significant efforts for establishing the fundamentals which comprise of the enhancement of the institute's education quality, assertion, and procedure for the certification. HEC has formulated an efficient method and approach towards the expansion and betterment of the education in the postsecondary institutes in the country. The procedure initiates by taking up the responsibility followed by the capacities which have to be conque4red for the achievement of the objective. The main objective of the framework for the quality assurance is to provide assurance that all the progress constitutes of the high level of quality, this is done by 
improving the research conduction procedures as well as the production of quality graduates. Moreover, the efforts made by the universities for the achievement of this task serve as the key role in the expansion of the nation's economy (Tovey, 2013).

\section{The Distance Education and ICT Associated Concerns:}

The term e-learning cannot be characterized as the distance; it is because the learner and the facilitators in it may not be physically separate. Therefore, the use of information and communication technology (ICT) in the educational programs doesn't provide enough evidence that these are utilized as the source for the distance learning. However, the information and communication technology is serving as the main source for the students in self-discovery and establishment of contacts with the professors and facilitators who are physically away in other continent or country. For achieving efficiency, the Open Distance Education makes use of the information and communication technology (ICTs)

The challenges of the open universities which offer the distance learning facilities have been increased because of the advancement of society pertaining to the awareness and internet presence. The prime strategy adopted for its eradication by the open learning institutes was to make a significant investment in designing the course pattern. This was supported by the fact that the incurred cost would be amortized with time.

\section{ICT and its Right to Use:}

The concern related to the quality assurance in education is not confined to the access of educational opportunities but also to the fact that how much assistance it can provide in improving the educational opportunities in terms of its success level. This point plays a more significant role when the implementation is related to the open educational institutes.

The incorporation of the advanced skills pertaining to the technology usage are assimilated in the learners by carrying out the certain actions. The foremost step is to integrate the technology which is properly functioning. There are various scholars in South Asia who have highlighted in their study that learning cannot occur when the connection is not available. This is applicable to those students who have not been 
provided with the learning gears. The second important factor is to let the user access the technology at a personal level to let him gain command over it. This fact has been highlighted in various studies but the most recent work is found in South Asia related to its Digital Education Enhancement Project (DEEP). Two universities have established this project, one is the University of Fort Hare located in South Asia and the other is located in the UK called Open University.

The number of Eastern Cape teacher who participated in it was 25 in which each was provided with palmtops. This was done so that they can easily use it, in a flexible manner in home and classroom as well. The third aspect is to provide the necessary training for the technology utilization, not only related to the basic but also use in the performance of the work-related task. This can be observed in the University of Pretoria's Centre for Applied and Augmentative Communication, an interdisciplinary course for masters. Although the participants are lecturers, It is essential that they are nurtured on the technology and are guided properly by the structure in their asynchronous online conversation. In addition, the DEEP project is centered to the functioning of the teachers. The training constitutes of nurturance on the surfing on the internet, curriculum pertaining to a specific subject along with the individuals and group use of the technology and web which aid in communicating the curriculum required for the professional job.

The fourth factor calls in the evaluation of the individuals. The study presented by SAIDE's related to the Multimedia Education Group of the Cape Town University, suggests approaches for monitoring how much the technological intelligence has been developed in the student. For instance, the word processing nurturance of the student can be reflected in his procedures word file where he has applied all the techniques and formatting along with other things. In many cases, the good to be used is same; however, the mechanism may vary. Moreover, the evaluation of the student's technological capacity assists in determining the student's ability to reflect and classify things. In the present era, the copy and paste trend in at rising because of the smooth format, however, this copy paste capability undermines our effort for corresponding the context of the text as well as logic, the present era also calls in long documents as it is believed to increase the impressiveness. This practice has increased the dissertation need. 
The fifth factor involves the constant analysis of the influence the technology and various advanced software's have on the teaching capability and learning competency of the students. The thing that is considered to be imperative is to consider the learning and teaching advantages related to the technological and advancement rather the adopting technology for the teaching and learning which results in unseen difficulties and problems. This is evident from the Open University and Leeds University research which was concentrated on the analysis of the virtual learning circumstance (Whitelock et al., 2000).

The supposition with regard to this is that the technologically rich environment improves the educational learning ability. However, this is contrasted with the Open University research which found out that it was the amalgamation of the web-based programs which assisted in nurturing the student on the various biological compositions as well as processes pertaining to the cognitive development. The virtual educational environment that is interactive in nature requires experiencing as well as theorizing. This real interference developed students thinking ability.

Lastly, the management is required to undergo training pertaining to the conceptualization of the skills as well as technology which then assist in conquering the exponential leaps that existed as well as the efficient performance of the task. The SAIDE is a function with institutes for the execution of the management system devised for distance learning, this elucidates that the information requirement nurturance is not only limited to the technology but is associated with the assistance that technology provides for managing the information in various ways. Moreover, the information system is required to not only be designed carefully but also to be inconsistency with the population, along with proper organization and the generation of data in a pattern which provides new information on the management of the educational programs.

\section{RESEARCH METHODOLOGY}

The approach adopted in the research is descriptive in nature, which uses a quantitative form of data derived from the close-ended aspects of the tool. The tool adopted is a questionnaire, in which the open-ended questionnaire is used for the production of the data in the qualitative form. The coding characterization determined for 
the qualitative data collection was determined according to the questionnaire item. The mechanism adopted include initial studying of the gathered data, after which they are arranged in sequential numbers after which the data is evaluated for studying the uniform pattern in correspondence to the items in the questionnaire.

After this, the uniformities patterns were written in descriptive form. The code categorization took place by utilizing words and phrases, which were also utilized for organizing the generated data. This assures that the answer pertaining to the question is separate from the other data. The data is then illustrated in the form of graphs by percentages and frequencies.

\section{Population and sample:}

The population of the study belongs to the multiple disciplines in the department which are part of Pakistan's federal institutions of Higher education in Islamabad. The respondents of the research are students, chairmen, along with chairladies which were part of these four educational institutes:

- National University of Modern Languages,

- Allama Iqbal Open University,

- National University of Science and Technology

- Bahria University.

The research samples were selected by the investigator, who applied the purposive sampling techniques. The sampling techniques rationale in qualitative nature is determined by the Creswell who states that the research purpose is not limited to categorizing the population but for the development of the inclusive research based on a central phenomenon that is attained by implementing the purposive sampling technique. The sample size of the students is 40 and the departmental heads are 21 .

\section{Data collection:}

The data was collected by a questionnaire which used both open-ended and close-ended questions, which analyzed four aspects such as:

1. The level of quality development unit in Pakistani Universities.

2. The problems in the quality education assertion and enhancement.

3. The present institute concerns of quality assertion and augmentation. 
4. The expected difficulties to be faced by the institution of higher education in accessing information on quality assurance and enhancement.

\section{Data Analysis Technique:}

The SPSS tool was adopted for evaluating the quantitative data. The comprehensiveness of the developed questionnaire was studied upon data compilation and before it as well as the data coding. The data were categorized according to the set objectives. After which the data was studied using percentages and frequencies.

\section{Results:}

The responses of the participants are provided in the chart below. The data is explained in the form of the self-exploratory percentages and frequencies. The main aspects studied in the research include the level of quality development unit in Pakistani Universities, the problems in the quality education assertion and enhancement, present institute concerns of quality assertion and augmentation, and the expected difficulties to be faced by the institution of higher education in accessing information on quality assurance and enhancement.

\begin{tabular}{|c|c|c|}
\hline \multicolumn{3}{|l|}{ Level of Quality Development Unit in Universities } \\
\hline & $\mathrm{F}$ & $\%$ \\
\hline The management assistance to quality assertion program & 9 & 42.86 \\
\hline Management commitment towards quality education & 11 & 53.38 \\
\hline The effectiveness of QEC system & 12 & 57.14 \\
\hline The synchronization and connection among departments & 12 & 57.14 \\
\hline
\end{tabular}

Chart 1: HOD answer on Level of Quality Development Unit in Universities

The illustrated data provides the evidence that majority i.e. $42.86 \%$ Departmental heads have a conviction that management assists in the quality assertion program, $53.38 \%$ believes that management is a commitment towards the provision of quality education. Furthermore, the response to the effectiveness of QEC system along with synchronization and connection among departments is believed to be $57.14 \%$. While the student's response to the management assistance in the quality assertion program is $60 \%$ which is same as on the effectiveness of QEC system. Moreover, the students who 
believe the management is a commitment towards the provision of quality education is $60.50 \%$. The information is summed up in chart 2

\begin{tabular}{|l|c|c|}
\hline & F & $\%$ \\
\hline Management assistance in the quality assertion program & 24 & 60.00 \\
\hline Management commitment towards the provision of quality & 25 & 62.50 \\
\hline The effectiveness of QEC system. & 24 & 60.00 \\
\hline
\end{tabular}

Chart 2: Students' answer on the Level of Quality Development Unit in Universities

\section{Education Quality and its Enhancement Problems:}

Considering the quality assurance in education, $71.43 \%$ departmental head belief that HEC ensures the sustainment of the institute's vision. The belief that HEC makes certain the quality education outline is $52.38 \%$ and that the staff is nurture on the centers pertaining to quality assurance. Moreover, $42.86 \%$ of HOD holds the view that faculty members are aware of the quality objectives. The responses are summed up in chart 3

\begin{tabular}{|l|c|c|}
\hline & F & $\%$ \\
\hline Institutional vision is sustained by HEC & 15 & 71.43 \\
\hline HEC makes certain the quality education outline & 11 & 52.38 \\
\hline Staff is nurture on the centers pertaining to quality assurance & 11 & 52.38 \\
\hline Faculty members are aware of the quality objectives. & 9 & 42.86 \\
\hline
\end{tabular}

Chart 3: HOD answer on education quality and its enhancement problems

Chart 4 demonstrates that $65 \%$ of the students believe that Institutional vision is sustained by HEC. 55\% of the students hold the conviction that students are aware of the quality objectives, also $50 \%$ of the students hold the view that HEC makes certain of the quality education outline. Lastly, $45 \%$ said that students are nurture on centers pertaining to quality assurance.

\begin{tabular}{|l|c|c|}
\hline & $\mathrm{F}$ & $\%$ \\
\hline Institutional vision is sustained by HEC & 26 & 65.00 \\
\hline HEC makes certain the quality education outline & 20 & 50.00 \\
\hline Students are nurture on centers pertaining to quality assurance & 18 & 45.00 \\
\hline Students are aware of the quality objectives. & 22 & 55.00 \\
\hline
\end{tabular}

Chart 4: Students' answer on education quality and its enhancement problems 


\section{Present Institute Concerns of Quality Assertion and Augmentation}

Chart 5 sums up that how much the educational institute's infrastructure, misunderstanding regarding work problems, difficulties in human resource management and limited funds in QEC impact the quality assurance of institutes.

\begin{tabular}{|l|c|c|c|c|}
\hline & HOD & \multicolumn{2}{l|}{ Students } \\
\hline & F & $\mathbf{\%}$ & F & \% \\
\hline Inappropriate infrastructure (ICT) & 12 & 57.14 & 25 & 62.50 \\
\hline The misunderstanding regarding work problems, & 13 & 61.90 & 24 & 60.00 \\
\hline Difficulties in human resource management & 12 & 57.14 & 26 & 65.00 \\
\hline Limited funds in QEC & 12 & 57.14 & 28 & 70.00 \\
\hline
\end{tabular}

Chart 5: Students' and Heads of Department answer on Present Institute Concerns of Quality Assertion and Augmentation

\section{Expected Difficulties of Higher Education on Quality Assurance and Enhancement}

Graph 1 underneath presents that departmental heads view $51.14 \%$ and students 75.00\% suggested that Truthful academic study be expected Difficulties of Higher Education on Quality Assurance and Enhancement, while 51.58\% and 48.42\% of heads of Department and students respectively regard maintaining standard as a major challenge. Lastly, $40.1 \%$ of Departmental head and 59.9\% of students believe that contribution made by the teachers and student ts as the major challenge in future.

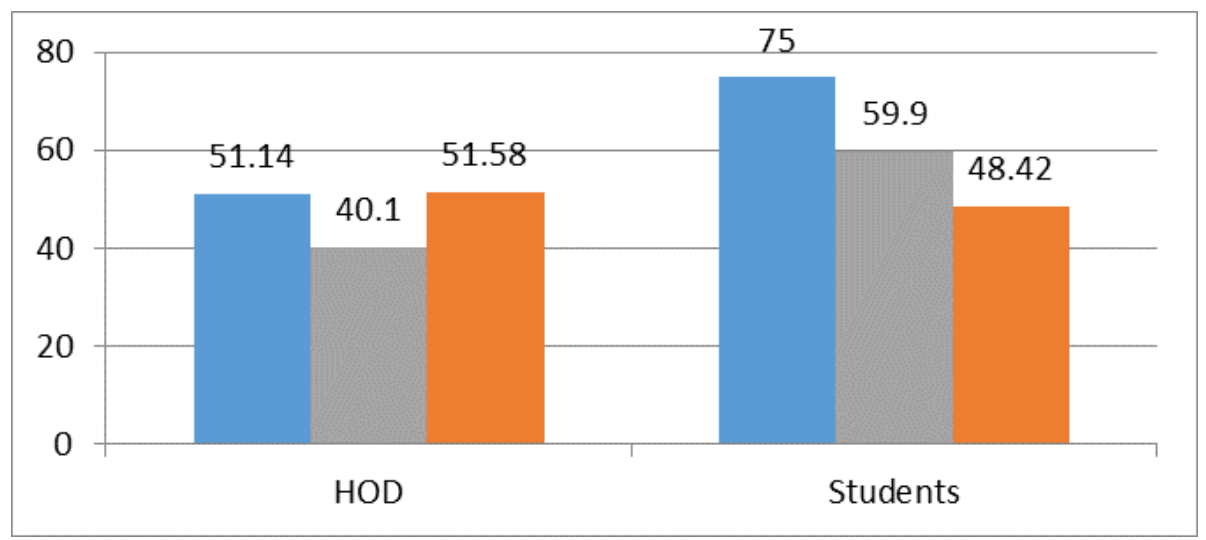

Graph 1: Expected Difficulties of Higher Education on Quality Assurance and Enhancement

\section{FINDINGS AND CONCLUSION}

Concerning the difficulties pertaining to the quality assurance and improvement, the participant responded that there are units in educational institutes for improving the 
quality of education provided, however, and the majority is not nurtured on them. The students and staff are not provided with the necessary guidance and the consultant. Furthermore, the answers show that the staff is not nurturing in the policy that institute has formulated for the quality assurance. Majority awareness level is low because of the open standards and the policy. The university is required to nurture on the fundamental that the quality is a constant process, which requires the stakeholders and the associated to work together in order to achieve the set goals. The actual situations and scenario contribute towards it as that quality requires commitment, persistence, and consistency. Furthermore, concerning the level of quality development unit in Universities, most of the departmental heads believe that the units are efficient, as they don't have awareness on the main functioning of the cells, while the student's quality improvement units were realized by their work's Turnitin report. HOD and students both concurred that the units for quality improvement are functional for the university sake of quality. The answers gathered from the Allama Iqbal Open University illustrate that the outline for quality enhancement cannot be applied to the formal universities. Currently, the HEC is required to take steps towards the disintegration of the standards set for the quality improvement and assurance by integrating Open Distance Learning Institutions stakeholders. Lastly, it is the duty of the HEC to form a policy for the educational institute's quality improvement and assurance. Moreover, a different policy is desired for the institutes who provide distance education as their provision of education varies from the paradigm form of the educational institutes.

\section{RECOMMENDATIONS}

1. The need here is to formulate a distinctive quality enhancement and assurance method for the Universities providing distance education as their deliverance method is different.

2. The quality assurance and improvement in the educational institutes are said to be comprised of five pillars namely attainment of the quality and the standards set, assistance to the individuals involved, improving the educational outcomes 
pertaining to quality, integration and autonomous aspect as well as nurturance on the global perception of quality.

3. The HEC is required to improve the QEC effectiveness by increasing its expenditure for improving the educational institutes quality.

4. For the improvement of the policy, the discussion must be held with all the stockholders regarding the policy.

5. The students should be nurtured on the quality performance increase of the educational units and that their concerns must be taken into regard when devising a policy.

\section{REFERENCES}

Batool, Z and Qureshi, R.H. (2006). Good Practices for Quality Assurance for Accreditation councils in Pakistan, Higher Education Commission, Islamabad.

Brunetto, Y., \& Farr-Wharton, $R$ (2005). Academics' responses to the implementation of quality Agenda. Quality in Higher Education, 11(2), 161-180

Bureau, E. (2009). Good Practices in Quality Assurance: A Handbook for the Sub-degree Sector. Education Bureau/Hong Kong Council for Accreditation of Academic and Vocational Qualifications/Joint Quality Review Committee.

Daniel, J. S. (1996). Mega-universities and knowledge media: Technology strategies for higher education. Psychology Press.

Distance Education and Training Council (DETC) (1998). Distance Education Survey. Washington $D C: D E T C$.

Glossary, E. Q. (2010). Developed by Unit B1" Quality. Classifications", Eurostat.

Hallak, J. (2006). Governance in education: transparency and accountability. M. Poisson (Ed.). UNESCO, International Institute for Educational Planning.

Harvey, L. and Green, D. (1993).Defining "quality assessment and evaluation in Higher Education." 18(1), 9-34

Higher Education Commission. (2009). Higher Education Commission Report 2002-2008.

Husain, I. (2007). Social sciences receiving due attention. HEC News and Views, a Monthly Magazine of Higher Education Commission.

ICAR (2002). Hands-on Training Manual on Peer review for Accreditation of Academic institutions and Programs, New Delhi: ICAR

IHEP (2000). Quality on the line: Benchmarks for Success in Internet-based Distance Education, Washington IHEP.

Inglis, A. (2005). Quality improvement, quality assurance, and benchmarking: Comparing two frameworks for managing quality processes in open and distance learning. The International Review of Research in Open and Distributed Learning, 6(1).

lqbal, A. (2004). Problems and prospects of Higher Education in Pakistan.University of Arid Agriculture, Rawalpidi.

Jung, I. (2005). ICT-pedagogy integration in teacher training: Application cases worldwide. Educational Technology \& Society, 8(2), 94-101.

Kalam, A. (2003). Attempting for excellence in higher education. Paper presented at National Conference on Quality Assurance in Education in Pakistan. Pakistan institute of Quality Control, Lahore

Khawaja, A.H. (1996). Difficulties and possibilities in University, Pakistan Perspective, vol. 1, No. 3. University of Karachi, Karachi

Lemaitre, J.M. (2008). Impact of quality assurance processes in higher education institutions.Quality assurance in Higher Education.HEC-printing press. 
Lenn, M. P. (1992). 11 The US Accreditation System. In Quality assurance in higher education: proceedings of an international conference Hong Kong, 1991 (p. 161). Routledge.

Marginson, S. (2002). Quality Assurance for Distance Learning: Issues for International Discussion and Action, CHEA International Seminar III on Academic Quality: Policy, Preferences and Politics. San Francisco, January 24.

Mohanthy, J. (2000). Current trends in higher education. New Delhi India: Deep \& Deep.

Mukhopadhyay, M. (2005). Total Quality Management in Education, 2nded, New Delhi: Sage

Nigvekar, A.S. (1996). Quality assurance in higher education: An Indian dilemma, journal of Higher Education, 19 (3), 353-364.

Nordic Recognition Information Centres (2006). Information on recognition of foreign qualifications in the Nordic ENIC/NARIC offices.

Prasad, V.S. (2005). Value Framework for Assessment of Higher Education Institutions, Bangalore: NAAC

Quality Assurance Agency (QAA) (2002). Distance Learning Guidelines, online www.qaa.ac.uk

Stella, A., \& Gnanam, A. (2004). Quality assurance in distance education: The challenges to be addressed. Higher Education, 47(2), 143-160.

Tovey, P. (2013). Quality assurance in continuing professional education: An analysis. Routledge.

Whitelock, D., Romano, D., Jelfs, A., \&Brna, P. (2000). Perfect presence: What does this mean for the design of virtual learning environments? Education and information technologies, 5(4), 277-289. 